\title{
Plan de cuentas bajo las normas VEN NIF PYME en las administrativas contables
}

\author{
Chart of accounts under VEN NIF PYME standards in administrative accounting \\ Plano de contas sob os padrões VEN NIF PYME em contabilidade administrativa
}

\author{
《 María Colmenares \\ mariamdzein@gmail.com \\ ORCID: 0000-0002-5989-8519 \\ Universidad de Carabobo, Venezuela
}

\author{
《 Anderson Mendoza \\ anderson.mendoza.26@gmail.com \\ ORCID: 0000-0003-0276-7750 \\ Universidad de Carabobo, Venezuela
}

\author{
《L Luis Moreno \\ luisemorenoe44@gmail.com \\ ORCID: 0000-0002-0996-4776 \\ Universidad de Carabobo, Venezuela
}

Recibido: mayo 2019 / Aceptado: junio 2019 / Publicado: septiembre 2019

\section{RESUMEN}

El objetivo de esta investigación se centra en diseñar un plan de cuenta bajo las normas VEN NIF PYME que oriente la toma de decisiones administrativas - contables al sector ganadero venezolano, que les permita recopilar, clasificar y presentar la información financiera bajo los parámetros establecidos en dicha normativa y que al compilarla en los estados financieros, pueda ser comparable a nivel global, satisfacer las necesidades de los usuarios pero sobre todo brindar a la gerencia la información suficiente para la toma decisiones que estos consideren asertivas para el desarrollo del sector. Esta investigación se desarrolló bajo un modelo de proyecto factible de carácter descriptivo en base a comprensión de la realidad de las transacciones de las industrias bovinas para poder desarrollarlo.

Palabras clave: Industria bovina; normas de Información financiera; contabilidad, plan de cuentas

\section{ABSTRACT}

The objective of this research is to design an account plan under the Ven NIF SME standards to guide administrative and accounting decision-making in the Venezuelan livestock sector, allowing them to collect, classify and present financial information under the parameters established in said normative and that when compiled in the financial statements, it can be globally comparable, meet the needs of users but above all provide management with enough information to make decisions that they consider assertive for the development of the sector. This research was developed under a descriptive feasible project model based on an understanding of the reality of the transactions of the bovine industries in order to carry it out.

Key words: Bovine industry; Financial Reporting Standards; accounting, chart of accounts
RESUMO
O objetivo desta pesquisa está centrado na concepção de um plano de contas sob os padrões Ven NIF Pyme que orienta a tomada de decisões administrativas - contabilidade para o setor pecuário venezuelano, que permite coletar, classificar e apresentar informações financeiras sob os parâmetros estabelecidos no normativo e que quando compilado nas demonstrações financeiras, pode ser comparável a nível global, satisfazer as necessidades dos usuários, mas acima de tudo fornecer à administração informações suficientes para tomar decisões que considerem assertivas para o desenvolvimento do setor. Esta pesquisa foi desenvolvida sob um modelo de projeto factível de natureza descritiva a partir do entendimento da realidade das transações da indústria bovina para desenvolvê-la.

Palavras-chave: Bovine industry; Padrões de relatórios financeiros; contabilidade, plano de contas 


\section{INTRODUCCIÓN}

$\mathrm{E}$ I sector primario de la economía ha tenido un papel fundamental en el desarrollo del ser humano, pues desde sus etapas más primitiva el hombre dependía completamente de los recursos naturales de su entorno, se alimentaba de los diferentes frutos que le proveía la naturaleza; cazaba bovinos, cérvidos, peces y otros animales para servirse de su carne, cuero y demás.

Tanto así, que con el pasar de los años el desarrollo del hombre y las civilizaciones se basó en formas para mejorar y aprovechar aún más a este sector, un ejemplo de esto es que ya desde la etapa neolítica el hombre comenzó a domesticar animales y a desarrollar el uso de la agricultura para así poder cubrir sus necesidades con mayor certidumbre y menor esfuerzo.

Por lo tanto, el sector ganadero está vinculado con la satisfacción de las necesidades de los seres humanos, tanto en el ámbito económico como social y considerando el contexto latinoamericano actualmente es posible observar cómo los sectores de la economía se han desarrollado y las necesidades del hombre han ido diversificándose de manera exponencial desde la conquista española iniciada en 1492, así como también han surgido cambios sustanciales en la producción y consumo de bienes. Sin embargo, la ganadería, en especial la bovina es un rubro del sector primario con gran potencial a nivel regional y nacional por las características geográficas y climáticas de América Latina, no solo para el consumo de carne o uso del cuero sino como materia prima para los demás sectores de la economía nacional o extranjera por sus grandes ventajas comparativas con otras partes del mundo.

En este marco de ideas, a nivel nacional, y desde un aspecto económico, los indicadores no son favorables para el desarrollo de la industria ganadera por un conjunto de factores; entre los más notables se tiene: la alta inflación, fijación de precios por parte del estado, continuos aumentos del salario mínimo, dificultad para conseguir productos importados. Un ejemplo de esto son las cifras que presenta el Instituto Nacional de Estadísticas (INE) en las cuales se evidencia como el país pasó de gastar 10.380.351 de dólares en importación de carne en 2003 a 1.976.631.858,93 de dólares en 2013, lo que representa un incremento del 19.042\%.

De allí que, las empresas del sector de la ganadería están obligadas a emplear herramientas que permitan adecuar y ajustar los procesos productivos de manera eficaz y eficiente para poder mantenerse y crecer dentro de una economía y una sociedad tan convulsionada, que ahora más que en cualquier otro momento requiere fortalecer la soberanía alimentaria.

Ahora bien, la necesidad de establecer procesos de calidad en todas las áreas que comprenden las operaciones de la industria ganadera depende en gran medida del registro, integridad y fiabilidad de la información que se deriva de las mismas. Información que para cumplir con esos requisitos debe ajustarse a los Principios de Contabilidad Generalmente Aceptados (PCGA) que permiten estandarizar las técnicas contables para registrar la información que generan los procesos productivos de manera adecuada, sin embargo, por su amplitud en algunos países es necesario 
ajustar los principios a las realidades económicas de cada país conservando la esencia de los mismos.

Por lo tanto, la forma apropiada para ajustar la información financiera a la normativa vigente es a través de un plan de cuenta que permita registrar todos los datos que se originan a diario en las diferentes áreas y etapas del proceso productivo desde el nacimiento de las crías hasta su venta según los parámetros contables establecidos. Es por ello, que estos datos cobran vital importancia pues de ella se derivan toda la información necesaria para tomar decisiones, establecer precio de venta, valoración de inventarios, analizar el movimiento de las cuentas reales y nominales de la industria, entre otros.

En una entrevista realizada a Jiménez, integrante de la directiva de la Asociación de Ganaderos del Estado Carabobo, se le preguntó si las organizaciones dedicadas a la producción de carne vacuna estaban registrando las operaciones bajo las Normas Internacionales de Información Financieras aplicables en Venezuela, quien señaló que existen algunas compañías muy organizadas que manejan programas contables, aunque por lo general la mayoría de ellas desarrollan sus operaciones de manera tradicional basándose en planificación y presupuestos; lo que ha originado la carencia de modelos contables, la utilización ineficiente de los recursos productivos, des favorecimiento de la industria y la carencia de visión gerencial que debe guiar a cualquier empresa.

En esta perspectiva, las Pequeñas y Medianas Empresas (PYME) dedicadas a la ganadería son una gran fuente de empleos en las zonas rurales del país que por lo general tienden a ser empresas familiares con registros bastantes rudimentarios que no disponen de las habilidades técnico-administrativas, donde las políticas contables se emplean de forma tradicional y no hay manuales y procedimientos establecidos que soporten el control interno, que si poseen las grandes empresas para mitigar posibles riesgos, hacerle frente a los continuos cambios de la economía y puedan llevar el ritmo que la competitividad global genera. De esta forma pueden mantenerse actualizadas en los sistemas de información de los procesos contables, es por ello que Mazza (2004), expone que "la información y la tecnología tienen un rol cada vez más estratégico, para administrar operaciones, mejorar la eficiencia y obtener ventajas competitivas en mercados que cambian rápidamente" (p. 11).

Adicionalmente, se evidencia en la práctica profesional del contador público venezolano que revelan la información financiera de las Pequeñas y Medianas Empresas (PYME), de esta índole, que no manejan un plan de cuenta específico para este sector, lo cual es una de las dificultades principales para que estas entidades presenten la información financiera adaptada a lo que las VEN-NIF PYME establece.

Por consecuente, los métodos mediante los cuales se registra y clasifica la información contable de las PYME dedicadas a la ganadería tampoco están adecuados a las normas y métodos establecidos para el país. Lo anterior genera incoherencias contables entre el marco regulador establecido por el ente rector de la materia y la información presentada por las PYME ganaderas 
que no permite el uso eficiente de los datos generados en los procesos productivos para la toma de decisiones.

Cabe destacar, la ganadería bovina en Venezuela posee gran potencial de explotación por sus grandes ventajas comparativas con otras partes del mundo, sin embargo, para el desarrollo de la misma se depende del registro, integridad y fiabilidad de la información que de allí se deriva, por lo cual este trabajo de investigación, tiene como objetivo central, diseñar una herramienta contable para las entidades del sector ganadero que refleje fielmente las actividades productivas del mismo. Con la finalidad de proporcionar a la gerencia de estas entidades, información financiera óptima para la toma de decisiones asertivas y que esta pueda ser comparable con entidades del mismo sector a nivel global, además de contribuir al desarrollo de la industria ganadera necesario para el progreso del país, y la calidad de vida de los venezolanos.

\section{Marco teórico}

\section{Procesos productivos ganaderos}

El proceso productivo ganadero es aquel mediante el cual las entidades relacionadas con la actividad desarrollan sus operaciones durante años con el fin de generar lucro mediante la producción de carne bovina, este comprende tres etapas fundamentales: la cría, el levante y la ceba del ganado bovino, aunque en ocasiones los ganaderos se orientan o especializan en una fase del proceso de producción del sector. Cabe destacar que no todos los ganaderos realizan todo el ciclo productivo del ganado, en algunos casos sólo se dedican a una fase del proceso productivo, ya sea cría, levante o ceba. A continuación, se sintetizan cada una de las etapas de la producción ganadera:

Partiendo de la fase inicial, la cría va desde el proceso de reproducción hasta entre los 6-9 meses de edad del ternero. Esta parte del ciclo productivo del ganado, tiene una línea de tiempo comprendida entre los 9 meses de gestación y entre 6 a 9 meses de alimentación. Al cuarto mes de vida del ternero es destetado, es decir, se interrumpe el suministro de leche materna para ser nutrido con aquellos alimentos que el productor ganadero considere apropiado. Dentro de esos alimentos están los pastizales, suministros vitamínicos y todos aquellos considerados necesarios para la crianza y crecimiento esperados del animal por parte del productor. Por lo anteriormente señalado, para la cría de los terneros es necesario la disposición de grandes extensiones de terrenos, mano de obra y gran disponibilidad de tiempo, por lo que es la fase menos atrayente para los ganaderos, además que el flujo del efectivo de la entidad disminuye debido a las grandes erogaciones de capital ya sean por servicios veterinarios, alimentos u obtención de nuevos especímenes para la reproducción de nuevas crías. Todas estas operaciones generarán rentabilidad a mediano plazo.

La segunda fase es la del levante, que consiste en el engorde de los terneros destetados tanto machos como hembras, a partir de los siete meses de edad hasta aproximadamente año y medio o 
dos años, siempre y cuando no superen los 230 kilogramos. Lo que se busca en esta fase del proceso productivo es llevar al ternero a un peso aproximado de $350 \mathrm{~kg}$ dependiendo de las exigencias del mercado, calidad alimenticia y raza del animal, para así llevarlos a la última fase que es la ceba, o en su defecto para la venta de éstos animales. Esta etapa del proceso productivo es uno de los periodos más rentables para el ganadero porque una vez engordado a la res puede venderla a buen precio. Cabe destacar que el mantenimiento de la res es menos costoso y el engorde del ganado va de forma exponencial debido su crecimiento, sin necesidad de alimentarlos con pastos de excelente calidad.

Por último, está la fase de la ceba, que consiste en definir el peso final de la res que se estima entre los $450 \mathrm{~kg}$ y $470 \mathrm{~kg}$ cuyas edades van desde los 19 meses hasta los 36 meses, con el fin de la matanza del novillo una vez que este haya alcanzado el peso apropiado. Actualmente los ganaderos implementan sistemas para mejorar la producción en la etapa del engorde del ganado, estos van desde el mejoramiento de las pasturas, genética del animal que consiste en aparear reses de gran calidad genética y utilización de productos adecuados para un eficiente cuidado y desarrollo del ganado, con esto se busca satisfacer las demandas de los consumidores que exigen carnes de alta calidad. Esta fase de la actividad productiva es muy rentable dependiendo de la cantidad y calidad alimenticia proporcionada a la res, si es de buena calidad a corto plazo generará grandes movimientos de caja ya que el animal aumentará rápidamente su peso y con ello lograr adquirir nuevas reses para reiniciar el ciclo productivo.

Para el cumplimiento de este proceso productivo ganadero es necesario la participación de recursos naturales, financieros, tecnológicos y humanos que se relacionen en conjunto para obtener las salidas del proceso, haciendo referencia en lo último a la producción de carne bovina.

\section{Contabilidad ganadera}

El enfoque de la investigacion se basa en la implementacion de herramientas contables para el desarrollo del sector ganadero. De esta manera, Gómez (1994), indica que:I

Es una rama de la contabilidad general que se asemeja a la contabilidad de costos, especializada en determinar, controlar, analizar e interpretar las partidas de los costos involucrados en la cría, mantenimiento y venta de animales. En resumen la contabilidad ganadera debe proporcionar al ganadero la información necesaria para obtener la rentabilidad máxima de su inversión. (pag 187)

De este modo, la contabilidad ganadera está orientada a dar seguimiento, control, análisis y evaluación a todas aquellas partidas vinculadas con la cría de ganado, y todos aquellos desembolsos adicionales necesarios para el cuidado, mantención y acción final de venta o sacrificio de la res. El fin de ésta rama de la contabilidad general es el poder asignar precios de venta y lograr determinar las ganancias o pérdidas generadas durante un período económico y así, de ésta 
forma facilitarle a la gerencia de las compañías agropecuarias la toma de decisiones, basadas en la información proporcionada a través de esos informes.

\section{Bases legales}

En la necesidad de alcance de el objetivo de el estudio, se realizó una revisión a la Legislación Venezolana, donde se logro evidenciar que no existe un basamento legal suficiente y específico de la presentación de la información financiera de entidades económicas dedicadas al sector ganadero; solo de manera general hace referencia las siguientes:

\section{Constitución de la República Bolivariana de Venezuela (CRBV, 1999)}

El título IV del Sistema Socioeconómico, Capítulo I del régimen socioeconómico y de la función del Estado en la economía, artículo 305 de la contitución vigente referente a la agricultura, establece:

El estado promoverá la agricultura sustentable como base estratégica del desarrollo rural integral a fin de garantizar la seguridad alimentaria de la población; entendida como la disponibilidad suficiente y estable de alimentos en el ámbito nacional y el acceso oportuno y permanente a estos por parte del público consumidor. La seguridad alimentaria se alcanzará desarrollando y privilegiando la producción agropecuaria interna, entendiéndose como tal la proveniente de las actividades agrícolas, pecuarias, pesquera y acuícola. La producción de alimento es de interés nacional y fundamental para el desarrollo económico y social de la nación. A tales fines, el estado dictara las medidas de orden financiero, comercial, transferencia tecnológica, tenencia de la tierra, infraestructura, capacitación de mano de obra y otras que fueren necesarias para alcanzar niveles estratégicos de autoabastecimiento.

Éste artículo hace una referencia acerca de la obligación del estado en promover e invertir en el sector y la importancia de la agricultura para lograr el desarrollo socioeconómico del país.

\section{Código de Comercio (CC, 1955)}

El CC rige las obligaciones de los comerciantes en sus operaciones mercantiles y los actos de comercio, aunque sean ejecutados por no comerciantes.

En lo que respecta a la contabilidad, el CC menciona las siguientes disposiciones:

- Artículo 32: Todo comerciante debe llevar en idioma castellano su contabilidad, la cual comprenderá, obligatoriamente, el libro Diario, el libro Mayor y el de Inventarios. Podrá llevar, además, todos los libros auxiliares que estimará conveniente para el mayor orden y claridad de sus operaciones. 
- Artículo 44: Los libros y sus comprobantes deben ser conservados durante diez años, a partir del último asiento de cada libro.

Por otra parte, el ordenamiento jurídico venezolano en la Ley del Impuesto Sobre la Renta y la Ley del Impuesto al Valor Agregado, leyes pilares en la regulación del sistema tributario venezolano establece que se debe llevar de forma ordenada los registros bajo los Principios de Contabilidad Generalmente Aceptados; expresando lo siguiente:

Ley de Impuesto Sobre la Renta (LISLR, 2015)

En el título VII Del Control Fiscal. Capítulo I de la Fiscalización y de la Reglas del Control Fiscal, artículo 88 referente a la obligatoriedad de llevar los libros y demás registros financieros bajo Principios de Contabilidad Generalmente Aceptados en Venezuela, señala:

Los contribuyentes están obligados a llevar en forma ordenada y ajustados a principios de contabilidad generalmente aceptados en la República Bolivariana de Venezuela, los libros y registros que este Decreto con Rango, Valor y Fuerza de Ley, su Reglamento y las demás Leyes especiales determinen, de manera que constituyan medios integrados de control y comprobación de todos sus bienes activos y pasivos, muebles e inmuebles, corporales e incorporales, relacionados o no con el enriquecimiento que se declara, a exhibirlos a los funcionarios fiscales competentes y a adoptar normas expresas de contabilidad que con ese fin se establezcan.

Ley de Impuesto al Valor Agregado (LIVA, 2014)

Artículo 56: Los contribuyentes deberán llevar los libros, registros y archivos adicionales que sean necesarios y abrir las cuentas especiales del caso para el control del cumplimiento de las disposiciones de este Decreto con Rango, Valor y Fuerza de Ley y de sus normas reglamentarias.

\section{Bases normativas}

Normas Internacionales de Información Financiera para Pequeñas y Medianas Entidades (NIF-PYME).

Las Normas Internacionales de Información Financiera contienen todos los requerimientos necesarios para la presentación de estados financieros de las pequeñas y medianas entidades, registros adecuados de las diversas transacciones relacionadas con las entidades, independientemente del tipo de actividad en las que se desempeñen y adaptaciones a cambios surgidos en diferentes períodos contables. Además contienen fundamentos teóricos, necesarios 
para la clasificación y determinación de forma efectiva las transacciones ocurridas en las pequeñas y medianas entidades, con el fin de presentar información relevante que plasme de forma efectiva y congruente las actividades de éste tipo de entidades.

Por consiguientes, para la elaboración de un plan de cuentas que permita recolectar, resumir y clasificar las distintas transacciones de las pequeñas y medianas entidades del sector ganadero y que estas al presentarla sirva para facilitar la toma de decisiones, es indispensable la adopción de las Normas Internacionales de Información Financieras a fin de cumplir con los principios y normas establecidos por el gremio.

Normas de Información Financiera en Venezuela para Pequeñas y Medianas Entidades (VEN-NIFPYME) y Boletines de Aplicación en Venezuela (BA VEN NIF PYME)

Las Normas de Información Financiera aplicables en Venezuela para pequeñas y medianas entidades compuestas por las Normas Internacionales de Información Financiera (NIF) y en conjunto con los Boletines de Aplicación, son adecuaciones a la NIF emitidos por la Federación de Contadores Públicos de Venezuela que deben ser aplicados de forma obligatoria en desarrollo de las actividades productivas en las pequeñas y medianas entidades en Venezuela.

\section{MÉTODO}

$\mathrm{L}$ a Investigación fue de tipo descriptiva, la cual se desarrolló bajo un modelo de proyecto factible, debido a que se indagó acerca de los procesos productivos actuales y transacciones implementadas para el desarrollo de la industria bovina, adicionalmente describirá la normativa contable aplicada al sector ganadero y mediante esta se pretende diseñar un plan de cuentas bajo VEN-NIF que proporcione el registro de las operaciones y sirva para la toma de decisiones en la pequeña y mediana industria del sector ganadero.

Para el desarrollo de este estudio se aplicaron los instrumentos de recolección de datos para analizar la realidad que existe en el sector ganadero venezolano e indagar la factibilidad de diseñar un plan de cuentas bajo VEN-NIF-PYME dirigido a las pequeñas y medianas entidades del sector, se procedió a realizar el registro de la información recopilada haciendo uso de la estadística descriptiva, a objeto de algunas circunstancia de la investigación en las que se llevó a cabo un enfoque cuantitativo; esto con la finalidad de producir el cuerpo de conclusiones y recomendaciones alusivas a la temática tratada.

Por otra parte, se usó el método inductivo, el cual, permitió lograr el análisis cualitativo de la información en adición al método analítico o interpretativo, que fue utilizado para la comprensión de los datos recopilados mediante la aplicación de los instrumentos. 
Además, la población y muestra, estuvo conformada por ganaderos adscritos a la asociación de ganaderos del estado Carabobo y estado Yaracuy, por profesionales de la Contaduría Pública.

\section{RESULTADOS}

A través del análisis documental se estudió cada sección de las Normas de Información Financiera aplicables a las pequeñas y medianas entidades de Venezuela, específicamente la NIC 2 "Inventarios" y la NIC 41 "Agricultura".

En el mismo contexto se constató que la normativa cuenta con 35 secciones, de las cuales veinticinco (25) secciones sí se requieren para el diseño de un plan de cuenta dirigido al sector ganadero venezolano y solo diez (10) secciones no guardan relación; estadísticamente el estudio realizado demostró que cerca del $29 \%$ de las secciones que componen las NIF para PYME, deben ser aplicadas en algunos de los procedimientos contables de la entidad, las cuales son importantes para la presentación de la información, mas no poseen relevancia directa con respecto a la elaboración de un plan de cuentas para el sector ganadero bovino venezolano.

Así mismo, esta exhaustiva indagación arrojó que el $71 \%$ de la normativa es elemental para dar origen al diseño de un plan de cuentas bajo la normativa y poder brindar un método que permita unificar la aplicación de normas contables en las entidades del sector ganadero, de manera que sean globalmente aceptadas, comprensibles y que ayude a los inversores y participantes de los mercados de capitales del mundo a tomar decisiones.

De la misma manera, se procedió a interpretar las bases legales establecidas en el ordenamiento jurídico venezolano que regulen las actividades del sector ganadero, y establecen la formalidad de llevar registros contables. Se examinó el primer, segundo y tercer nivel de la pirámide de Kelsen, refiriéndonos a la constitución, códigos orgánicos y leyes respectivamente; posteriormente se llevó a cabo una observación en cada documento, a fin de encontrar normas que establecen el deber de llevar registros en la contabilidad de las distintas transacciones relacionadas con el pago de tributos, para ello se procedió a describir textualmente la norma, analizarla y adicionar aquellas cuentas necesarias al diseño del plan de cuenta del sector ganadero venezolano; entre tanto se estudió veinte (20) artículos que generalmente establecen el deber formal de llevar registros contables de las transacciones que una entidad del sector ganadero venezolano pueda mantener con la Administración Tributaria.

Cabe resaltar que se analizó el decreto presidencial de exoneración 2.287 que gozan las entidades explotadoras del sector pecuario venezolano, lo cual genera un tratamiento contable distinto al de cualquier otra entidad, debido a que maneja partidas en los estados financieros especiales por la subvención que el gobierno brinda mediante dicho decreto.

Así que, al interpretar las bases legales establecidas en el ordenamiento jurídico venezolano que regulan las actividades del sector ganadero, se desarrolló una serie de partidas a considerar en el plan de cuenta que revele las distintas transacciones que puede llevar a cabo una entidad 
ganadera con la Administración Tributaria, además del cumplimiento de los deberes formales establecidos.

\section{Propuesta. Diseñar un plan de cuenta bajo las normas VEN NIF PYME}

A través de la propuesta se pretende facilitar a las entidades del sector ganadero un plan de cuenta que este adaptado a las Normas Internacionales de Información Financiera aplicables a las pequeñas y medianas entidades en Venezuela.

\section{Presentación}

El sector primario de la economía desde la era neolítica hasta la actualidad ha sido un factor fundamental para el desarrollo de nuestras civilizaciones. Teniendo un papel esencial la ganadería bovina en Venezuela por sus grandes ventajas comparativas con otras partes del mundo. Sin embargo, para el desarrollo de la misma y establecer procesos de calidad en las áreas de la industria ganadera se depende del registro, integridad y fiabilidad de la información que de allí se deriva. Por lo que estas deben ajustarse a los Boletines de Aplicación de las Normas de Información Financiera en Venezuela para Pequeñas y Medianas Empresas que permiten registrar la información que generan los procesos productivos de manera adecuada; consecuentemente, la forma apropiada para ajustar la información financiera a la normativa vigente es a través de un plan de cuenta que permita registrar los datos que se originan a diario en las diferentes áreas del proceso productivo y que esto sirva de base para: tomar las decisiones que la gerencia considere asertivas para el desarrollo del sector

\section{Justificación}

En toda entidad económica, sea pequeña, mediana o grande, es necesario el control de sus actividades operativas, de financiamiento y de inversión que durante el tiempo va realizando, y solo lo podrá conocer a través de los indicadores que la información financiera le pueda suministrar; de esta manera se crea la necesidad de emisión de estados financieros que permitan compilar la información y suministrarla a los usuarios involucrados con dicha entidad, la cual al ser presentada debe cumplir con unos estándares y principios establecidos, que en el caso Venezolano nos referimos a los Boletines de aplicación que emite la Federación de Contadores Públicos de Venezuela por la adopción de las normas Internacionales de Información Financiera en el país.

Es por ello, que las entidades del sector ganadero venezolano no se escapan del cumplimento de estos principios contables para la presentación de sus estados financieros, y evaluando la situación actual son pocas las que cumplen con lo antes mencionado, de hecho, algunas llevan los registros de sus transacciones diarias de manera tradicional.

Por lo tanto, las entidades que conforman el sector ganadero venezolano, deben considerar la importancia de llevar sus registros contables adecuadamente y bajo las normas de información financiera establecidas, a fin de poder suministrar información optima a los usuarios involucrados, 
además de brindar ayuda a la gerencia a controlar sus costos, gastos y conocer la rentabilidad que el negocio les genera.

\section{Objetivos de la propuesta}

- Presentar un plan de cuenta al sector ganadero venezolano adaptado a las Normas de Información Financiera, que los oriente en la toma de decisiones administrativas-contables.

- Mostrar al sector ganadero el tratamiento contable que reciben los activos biológicos desde su nacimiento hasta que son considerados inventarios.

- Facilitar a la administración de las entidades del sector ganadero, las partidas a usar para el registro de gastos y costos que los procesos operativos generan.

- Diseñar las cuentas contables que la administración de la entidad debe considerar para la revelación de los ingresos ordinarios y extraordinarios que obtiene en el ejercicio económico.

- Permitir la comparabilidad de resultados entre distintos periodos lectivos, para la toma de decisiones en búsqueda del desarrollo y mejoramiento continuo.

\section{CONCLUSIÓN}

$\mathrm{L}$ uego del estudio de la investigación realizado sobre la contabilidad aplicada al sector ganadero a través de técnicas como observación y aplicación encuestas, se pudo constatar que la población que califica como PYME dedicada a este sector, existe precariedad en la aplicación de contable sobre sus registros y desconocimiento acerca del tema en cuestión, a pesar del vasto conocimiento que poseen los empresarios sobre cada detalle de las etapas del proceso productivo de la industria ganadera bovina.

Así mismo, el estado actual de las políticas contables utilizadas para el registro de las transacciones en el sector ganadero, existen puntos neurálgicos de divergencia con respecto a las consideraciones de los profesionales de la contabilidad sobre cómo debe aplicarse la normativa y emplear el tratamiento contable en una u otra transacción, es por ello que se afirmar que existen deficiencias notables en la aplicación actual de estas políticas.

Es válido destacar, que, si bien en líneas generales las entidades pro

.ductoras son similares, cada una define su manera particular de asumir cada proceso lo cual dificulta la determinación de un mecanismo compatible para el sector que ajusten cada particularidad de las diferentes entidades del sector ganadero a el deber ser contable. 


\section{REFERENCIAS}

Constitución de la República Bolivariana de Venezuela Gaceta Oficial No5098 Extraordinario del 19 de febrero de 2009

Código de Comercio Gaceta Oficial N475 Extraordinaria del 21 de diciembre de 1955

Decreto con Rango, Valor y fuerza $N^{\circ} 2287$, de la exoneración del Impuesto Sobre la Renta, sobre la explotación del sector primario

Decreto con Rango, Valor y fuerza de Ley de Impuesto Sobre la Renta en la Gaceta Oficial de la República Bolivariana de Venezuela $N^{0} 6210$ del 30 de diciembre de 2015

Decreto con Rango, Valor y Fuerza de Ley de Impuesto al Valor Agregado. Gaceta No. 38362 del 18 de noviembre de 2014

Decreto con Rango Valor y Fuerza de Ley No6072 de Ley del Régimen Prestacional de Vivienda y Hábitat
Decreto con Rango Valor y Fuerza de Ley N6266 de Ley del Seguro Social Obligatorio

Gómez, F. (1994). Contabilidad Ganadera: En Venezuela (Teoría y Práctica). Ediciones Fragor. Caracas, Venezuela

Normas Internacionales de Información Financieras para las PYME, pronunciamiento oficial 2015. Vigente a partir del 01 de enero del 2017

Norma Internacional de Contabilidad $N^{\circ} 2$ (NIC-2): Inventarios

Norma Internacional de Contabilidad N41 (NIC 41): Agricultura

Mazza, A. (2004). Escritos de tecnología de la información. (1ed). Argentina: Ediciones Cooperativas 